\title{
MONITORIZAÇÃO E CONSERVAÇÃO PREVENTIVA DE PATRIMÓNIO HISTÓRICO COM BIM: O PROJETO HERITAGECARE
}

\author{
Miguel Azenha ${ }^{(1)}$, Alberto Barontini (1), Daniel Oliveira ${ }^{(1)}$, Carlo Alarcón ${ }^{(1)}$, Hélder S. \\ Sousa ${ }^{(1)}$, Maria Giovanna Masciotta ${ }^{(2)}$ \\ (1) ISISE, Escola de Engenharia da Universidade do Minho, Guimarães, Portugal \\ (2) Departamento de Engenharia e Geologia (INGEO), Universidade "G. d'Annunzio", Chieti- \\ Pescara, Itália
}

\begin{abstract}
Resumo
A gestão de ativos (facility management) é uma área interdisciplinar que tem como objetivo manter um sistema e os seus respetivos serviços operacionais. Nesse contexto, as metodologias Building Information Modeling (BIM) já demonstraram ter capacidade de agregar e gerir informação na coordenação de processos, espaços, pessoas e atividades. No entanto, a definição de boas práticas de modelação e de estratégias para obtenção de informação para o caso de construções existentes, particularmente no caso do património histórico, é ainda um assunto em aberto. No presente trabalho apresenta-se uma abordagem viável para modelação, em plataforma BIM, da gestão de construções existentes com especial atenção para o acompanhamento da evolução de danos detetados em inspeções. A metodologia proposta requer a definição do nível de necessidade de informação adequado para o modelo e cada um dos seus componentes, baseado num equilíbrio entre características geométricas e não geométricas (ex. o nível de precisão no detalhe e a quantidade de informação acoplada). Para este efeito desenvolveu-se um procedimento que permite aos técnicos obter informação proveniente do modelo BIM para atualizar os cenários de dano, sendo baseado na interoperabilidade entre: (a) software BIM proprietário e open source, através do formato IFC (Industry Foundation Classes); (b) modelo BIM e folhas de cálculo. A metodologia desenvolvida é aplicada ao Paço dos Duques de Bragança, situado em Guimarães.
\end{abstract}

\section{Introdução}

A documentação e a preservação de edifícios existentes, particularmente edifícios com valor histórico, são atividades intrinsecamente multidisciplinares e envolvem diferentes abordagens, cada uma com os seus próprios conceitos, métodos e fontes de informação. Esta informação inclui normalmente componentes qualitativas (ex. imagens, desenhos, relatos orais, textos, entre outros) e quantitativas (ex. valores, intervalos, datas, índices, distâncias, entre outros). A natureza de tal informação é cumulativa, aumentando com o tempo e a disponibilidade de fontes de informação, e é, ao mesmo tempo, muitas vezes não existente ou desatualizada, devido a 
restrições de orçamento, falta de plataformas/meios adequados para recolher essa informação, restrições de tempo ou mesmo acessibilidade à informação em condições de ser armazenada. A gestão de ativos (facility management - FM) é uma área interdisciplinar que tem como objetivo uma administração eficiente de informação para manter um determinado sistema e seus respetivos serviços e componentes em operação com desempenho adequado.

Nos últimos anos, o Building Information Modelling (BIM) já deu provas de ser uma metodologia apta para suporte à gestão de ativos. A aplicação de metodologias BIM é relativamente expedita para a conceção e construção, já que o modelo é continuadamente atualizado durante este processo. No entanto, a sua aplicação para a documentação e gestão de edifícios existentes, especialmente património histórico e lugares arqueológicos, é complicada por lidar com informação esparsa e pela sua natureza inerentemente cumulativa. Em 2014, foi iniciado um esforço comum entre COTAC, ICOMOS, UNESCO, BuildingSMART, e outros participantes, levando à publicação de uma série de guias para o desenvolvimento de modelos para proprietários de ativos, utilizadores e profissionais [1,2]. Nesse âmbito, foi dada atenção específica a uma metodologia de trabalho adequada às particularidades das construções antigas. De acordo com os requisitos da série ISO 19650 [3,4], um dos importantes passos no planeamento de um modelo BIM consiste na definição, por todas as partes envolvidas, do nível de informação necessário (level of information need - LoIN) para o projeto. O LoIN pode ser decomposto em dois parâmetros: (1) nível de detalhe (level of detail - LoD) que define o quão precisa é a representação geométrica virtual de um objeto; (2) nível de informação (level of information - LoI) que define quão detalhada é a informação embebida no objeto virtual. A definição de um nível apropriado para cada elemento/componente é realizada em conformidade com o acordo estabelecido entre os intervenientes e baseado na importância desse elemento/componente, fontes de informação disponíveis, orçamento, restrições de tempo e, em geral, do objetivo do modelo [5]. Para o presente trabalho, o objetivo é a monitorização e gestão do ativo, com especial ênfase no apoio à caracterização de danos em edifícios existentes durante uma inspeção. Nesta área estão envolvidos dois tipos principais de profissionais, nomeadamente o gestor/modelador BIM e o inspetor. O gestor/modelador é responsável pelo desenvolvimento e atualização do modelo BIM. Neste âmbito, existem vários trabalhos publicados em que o BIM foi aplicado à preservação do património histórico, tendo forte enfoque num nível extremamente detalhado para a representação geométrica [6-9], que aumenta significativamente tanto o tempo como os custos associados ao desenvolvimento do modelo. Esta abordagem de extremo detalhe e acuidade geométrica tem sido também aplicada na modelação do dano encontrado durante operações de inspeção $[7,9,10]$. Outro caso é o uso de modelos "as-damaged" BIM de elevada precisão baseados em nuvens de pontos que foram utilizados para uma estratégia automática de avaliação pós-desastre [11].

O presente trabalho apresenta uma abordagem desenvolvida no âmbito do projeto de investigação HeritageCare (Interreg Sudoe) [12] para a modelação/identificação das patologias de construções históricas em plataforma BIM, tendo em consideração os problemas já descritos. Salienta-se que a modelação dos elementos compreende tanto a representação geométrica como a atribuição de propriedades e informação necessárias para a implementação de um sistema de gestão. Este trabalho é estruturado em duas partes principais, sendo a primeira parte a descrição da metodologia (capítulo 2) e a segunda referente à aplicação da metodologia ao Paço dos Duques de Bragança, Guimarães (capítulo 3). Por fim, as conclusões finais são expostas no capítulo 4. 


\section{Metodologia}

Na presente metodologia, o LoIN pretendido para o modelo é obtido através do compromisso entre a precisão dos pormenores e a quantidade de informação acoplada, almejando-se o equilíbrio entre um nível de exatidão (level of accuracy - LoA) baixo e alguns componentes com elevado LoI. Esta abordagem é coerente com a que já foi adotada em [2] para a modelação BIM de edifícios de valor histórico. Neste contexto, [5] introduziu o LoA como um atributo de cada elemento, tal como o LoD e o LoI, para apontar como a representação gráfica representa o estado real atual. Em alguns casos, é considerada necessária a representação detalhada do elemento construtivo, mas é-lhe associada uma precisão geométrica baixa. Na proposta aqui considerada, o responsável pelo desenvolvimento do modelo BIM terá de ter em mente as necessidades dos inspetores de forma a providenciar uma ferramenta prática e fácil de utilizar de forma a apoiar o seu trabalho. A representação gráfica tridimensional tem de ser suficientemente clara para identificar o elemento, a sua posição e que permita obter uma referência interna para rapidamente localizar um determinado dano. No entanto, o elemento em si não tem de ser reproduzido com fidelidade total à sua geometria real, neste contexto de gestão de inspeções, monitorização e planeamento de intervenções. Informação mais detalhada para efeitos de análise de evolução de danos podem ser incluídas, pois o modelo permite acoplar relatórios de inspeção e fotografias como repositórios de informação complementar. Tendo como exemplo a situação onde a inspeção de danos é feita a um bem móvel de um edifício histórico, nomeadamente uma estátua, a sua reprodução pode ser realizada eficazmente através de um cilindro localizado no sistema coordenado no seu exato local com uma aproximação aceitável às suas dimensões.

Dependendo do tipo, o dano pode ser modelado por representação explícita do mesmo, ou por representação simplificada (seja ao nível da simplificação gráfica da sua real configuração, seja ao nível de o representar apenas como informação não-geométrica). Na presente abordagem, optou-se sempre por representações simplificadas. Nesse sentido, a informação sobre danos globais que afetam todo o elemento construtivo (ex. deformação de uma parede) é alocada como uma informação não gráfica relacionada com o elemento em si. $\mathrm{O}$ dano que diga respeito apenas a uma parte do elemento construtivo (ex. sinais de humidade, fissuras, destacamento, corrosão, grafitti, entre outros) é graficamente modelado através de elementos "patch" de forma paralelepipédica, com espessura fixa $(1 \mathrm{~cm})$ sobreposta ao elemento construtivo e com dimensão análoga à envolvente do dano reportado. Esse elemento permite descrever a localização de um determinado dano, a sua classe, severidade e extensão.

De uma forma global, a abordagem que acaba de ser descrita é particularmente conveniente para edifícios existentes cujas características, em grande parte dos casos, são total ou parcialmente desconhecidas, pois permite reduzir o tempo e custos de aquisição de informação para a fase de modelação. A representação simbólica dos danos é considerada inequívoca e consistente. O modelo é desenvolvido através de um software proprietário (para este estudo adotou-se o Autodesk Revit), e pode ser convertido num formato aberto, nomeadamente o IFC. Durante o processo de inspeção no local, o inspetor pode facilmente aceder e interrogar o modelo utilizando um software de livre acesso (ex. Solibri Model Viewer ou BIMVision) e, como tal, a gestão desse ativo não requer a posse de uma licença específica de um software BIM. A interoperabilidade entre modelos BIM e folhas de cálculo permite uma rápida atualização dos resultados das inspeções. Aliás, cada componente do modelo é identificada por um código e toda a informação referente a este é listada através de um formato padrão inspirado 
no conceito de product data template - PDT. Os PDT são modelos para folhas de informação do produto (product data sheets - PDS) em formato eletrónico que, de entre outras utilizações, são usadas, no campo da produção de novos produtos, para proporcionar objetos BIM com homogeneização ao nível da informação embebível. Assim, os PDT são um formato estruturado que recolhe cada segmento de informação do produto numa forma consistente [13]. Neste trabalho, o conceito PDT foi adaptado às necessidades e especificidades de elementos arquitetónicos históricos. O formato PDT consiste numa folha de cálculo dividida em diferentes categorias de informação, cada uma com uma lista de parâmetros a preencher (ver exemplo aplicado na Tabela 1). A primeira secção do formato adaptado inclui, entre outros, o nome, o tipo de elemento, a sua adequabilidade de uso. A segunda secção providencia informação relevante para o estado de conservação do elemento, sendo esta dividida em seis categorias de parâmetros: (1) informação da construção (informação sobre datas de construção e intervenções); (2) informação de inspeções (informação de inspeções anteriores, referência do inspetor e datas); (3) informação dimensional (informação geométrica geral, principais dimensões); (4) informação estrutural dos elementos portantes (conjunto de propriedades mecânicas e informação estrutural); (5) caracterização de subelementos (parâmetros relacionados com partes constitutivas, diferentes materiais ou elementos, cada um com o seu próprio conjunto de parâmetros); (6) informação do nível global de dano.

Tabela1: Parte de PDT para uma parede duma construção histórica

\begin{tabular}{|c|c|c|}
\hline Parameter name & Value & Notes \\
\hline \multicolumn{3}{|c|}{ Masonry wall construction data } \\
\hline Construction date & Years & Example: 1500 \\
\hline $\begin{array}{l}\text { Construction date degree of } \\
\text { accuracy }\end{array}$ & 0 to 5 & $\begin{array}{l}0=\text { rough estimation without support, } 5=\text { totally sure }- \\
\text { Example: } 2\end{array}$ \\
\hline Previous intervention dates & Years & Example: $1590,1650,1900,2015$ \\
\hline $\begin{array}{l}\text { Intervention dates degree of } \\
\text { accuracy }\end{array}$ & 0 to 5 & $\begin{array}{c}0=\text { rough estimation without support, } 5=\text { totally sure- } \\
\text { Example: } 0,5,4,5\end{array}$ \\
\hline \multicolumn{3}{|r|}{ Inspection data } \\
\hline Inspection dates & Date & Example: $1985 / 07,1994 / 05,2010 / 05$ \\
\hline Last inspection reference & & Name, address or any other reference to track the source \\
\hline Brief description & & General description of last inspection \\
\hline Survey picture URL & & \\
\hline
\end{tabular}

Os elementos "patch" apresentam também o seu próprio formato PDT que recolhe a informação relevante. À semelhança dos elementos da construção, após uma primeira secção dedicada à recolha de metadados, os parâmetros do PDT para estes elementos são organizados em cinco categorias: (1) informação da classificação (classe e subclasses de dano tendo em conta o atlas de danos HeritageCare [12]); (2) informação de inspeções (informação sobre inspeções anteriores); (3) informação geométrica (informação sobre a presente e anterior aparência geométrica do dano); (4) indicações e diagnóstico (parâmetros que descrevem a severidade e intensidade do dano); (5) informação do controlo da evolução do dano (parâmetros que podem ser usados para controlar a evolução do dano ao longo das várias inspeções).

No local, os inspetores verificam os elementos e reportam numa folha de cálculo (desenvolvida para interoperabilidade no contexto da presente metodologia, mas acessível diretamente com 
Excel) qualquer informação nova que seja relevante. Depois, as folhas de cálculo são carregadas e enviadas para o gestor BIM que pode facilmente implementar a informação nova no modelo por importação direta de dados e, eventualmente, proceder a novas modelações de danos, se aplicável. Para mais detalhes sobre a metodologia aqui descrita, consultar a referência [14].

\section{Aplicação ao Paço dos Duques de Bragança}

\subsection{Descrição do caso de estudo}

O Paço dos Duques de Bragança em Guimarães é um monumento de elevado valor histórico e cultural no contexto português, sendo um dos monumentos mais visitados do país. A sua construção começou no ano de 1420, mas o edifício permaneceu incompleto e abandonado desde o início do século XVI, período no qual sofreu um prolongado processo de deterioração, destruição parcial e reutilização dos seus materiais para outros fins. Entre 1937 e 1959 foi realizada uma significativa intervenção, liderada pelo arquiteto Rogério de Azevedo, com o objetivo de restituir ao edifício o seu suposto aspeto original. A estrutura foi intervencionada e, em alguns casos, foram adicionados elementos de betão armado. Nesse contexto, foram adicionadas novas infraestruturas (ex. rede de saneamento, instalações sanitárias, rede de água e eletricidade), sendo que desde 1959 o Paço acolhe um museu.

\subsection{Estratégia de modelação}

A geometria global do Paços dos Duques foi inicialmente definida com base na informação existente, na forma de desenhos e plantas da reconstrução e outra documentação [15]. $\mathrm{O}$ trabalho de inspeção detalhada incidiu sobre a parte do edifício que contém os escritórios administrativos, tendo-se inspecionado 23 espaços para averiguar a fiabilidade das fontes de informação iniciais (Figura 1). Em cada compartimento foram feitas fotografias digitais aos elementos construtivos, incluindo fotografias panorâmicas ao espaço global. Nesse processo, foram identificados 36 elementos construtivos diferentes. Globalmente, as tarefas de inspeção demoraram cerca de 8 horas, sendo divididas em duas fases. A primeira foi maioritariamente relacionada com a identificação de elementos/compartimentos, enquanto a segunda fase foi dedicada à clarificação de dúvidas in-situ (ex. inspeção de janelas escondidas atrás de mobiliários, identificação de vigas de betão armado escondidas por revestimentos em madeira, entre outros). Durante a inspeção, os tipos de elementos foram agrupados tendo em conta o material de construção e a relação topológica entre os seus componentes. Para apoiar o desenvolvimento do modelo, foi criada uma lista de todos os elementos de cada compartimento. Para cada elemento foi definido um PDT tipo.

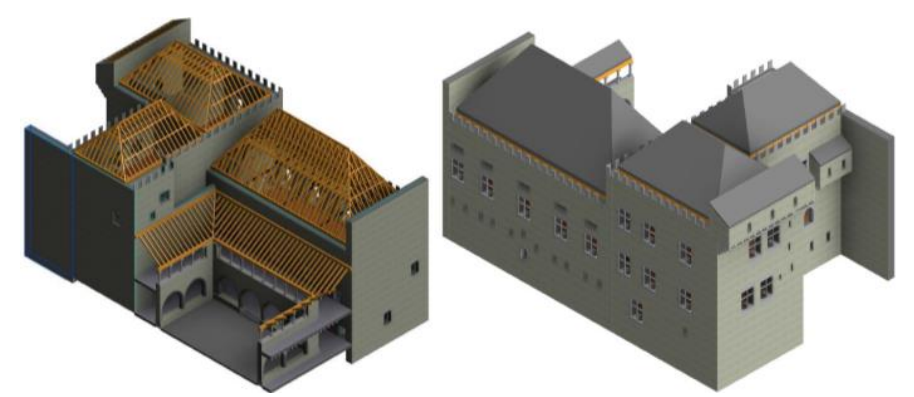

Figura 1: Isometria global do modelo BIM, com detalhe das coberturas de madeira. 
Após o processo de inspeção, foi realizada a representação da geometria num modelo numérico tridimensional e acoplada a informação de cada PDT a cada objeto criado. Esta tarefa pode ser dividida em dois passos. O primeiro consiste no desenvolvimento de classes de objetos, e o segundo passo refere-se ao objetivo de alocar cada elemento no modelo BIM em si, com especial atenção à relação espacial entre cada um. Foi criado um total de 25 classes de objetos BIM específicos para este modelo. Cada objeto é paramétrico e, como tal, o modelo foi criado somente uma vez para qualquer tipo de elemento e depois os parâmetros foram modificados de acordo com características específicas destes. A maioria dos elementos foi criada por hereditariedade, a partir duma classe de objetos nativa do software (ex. paredes, lajes, vigas de betão debaixo das lajes, entre outros). As classes de objetos que foram criadas incluem janelas, portas, arcos e colunas/pilares. A Tabela 2 apresenta alguns exemplos de elementos inspecionados com a correspondente representação geométrica virtual no software proprietário, utilizando componentes base de famílias de elementos encontrados em software de uso generalizado. De salientar que a simplificação realizada à forma geométrica dos elementos não deverá induzir à perda de identidade construtiva do elemento, apesar de ser necessário atender ao acordado entre as diferentes partes envolvias no processo de inspeção, modelação e gestão da estrutura para o nível informação necessário (LoID). A Figura 1 apresenta o panorama geral do modelo.

Por fim, a precisão do modelo foi avaliada através da comparação da geometria implementada, com base na documentação disponível e nos dados da inspeção, com os resultados de um varrimento laser da ala sul do edifício (feito posteriormente à primeira modelação BIM por condicionantes não controladas pela equipa de investigação). Esta tarefa demorou dois dias completos para o levantamento in-situ. Em cada compartimento, a localização do laser foi definida de forma a maximizar a quantidade de informação assim como no local de transição entre espaços de forma a ligar a informação de cada um destes, e de janelas abertas de forma a ligar o interior com o exterior do edifício. Foi utilizado um equipamento Leica P20 no levantamento e o ficheiro da nuvem de pontos originalmente de $40 \mathrm{~Gb} *$.pts foi processado pelo software BIM proprietário. O ficheiro final com cerca de 1164 milhões de pontos comprovou a precisão da geometria (ex. localização de janelas, inclinações e elevação dos elementos) com a geometria inspecionada (Figura 2) tendo, no entanto, sido encontradas algumas diferenças que permitiram retificar o modelo. Contudo, muitas dessas diferenças geométricas puderam ser negligenciadas considerando que o objetivo do modelo é atingir o nível pré-definido de informação requerida. Salienta-se que a metodologia proposta não toma como requisito necessário a utilização de varrimento laser, mas é suficientemente adaptável para assumir informação dada por essa ferramenta se disponível.

Tabela 2: Exemplos da representação de elementos em software proprietário.

\begin{tabular}{ccc}
\hline Nome & Fotografia da inspeção & Representação virtual \\
\hline Coluna/pilar & & \\
Janela em arco de pedra
\end{tabular}




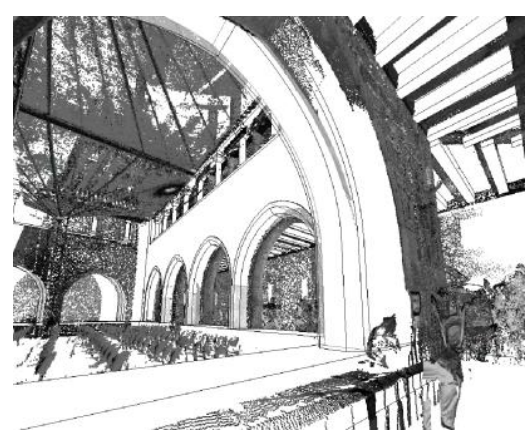

(a)

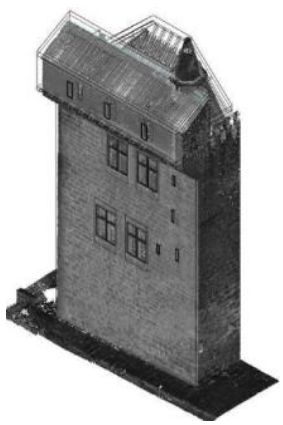

(b)

Figura 2: Modelo BIM e sobreposição da nuvem de pontos: (a) claustro; (b) parede exterior.

\subsection{Inspeção in-situ e levantamento dos danos}

A parede sul foi usada para testar a metodologia desenvolvida para implementar a informação referente às características dos danos existentes. A identificação de uma lista de danos oficialmente aprovada foi o primeiro passo para atingir uma uniformidade de representação. No presente trabalho, o atlas de danos desenvolvido no projeto HeritageCare [12], foi considerado como base para definir seis classes de dano, cada uma delas dividida em vários tipos de dano: 1) colonização biológica; 2) descoloração e depósito; 3) perda de material; 4) destacamento; 5) fissuras; 6) deformações. A última classe é referente à deterioração que afeta todo o elemento (ex. deformação no e fora do plano, encurvadura, inclinação, deformação excessiva e encurvadura). Assim, de acordo com metodologia adotada neste trabalho para modelação de danos, estas são descritas dentro de uma lista de atributos no PDT de cada elemento. No presente trabalho, os cenários identificados foram referentes a colonização biológica e fissuras. Foi definida uma escala de cores para este caso de estudo em específico: 1) verde-claro para podridão; 2) verde-escuro para micro-organismos; 3) azul para infestação de pragas; 4) amarelo para fissuras indefinidas; 5) vermelho para fissuras de caráter estrutural. Seguindo a presente abordagem, a fase de modelação é muito mais rápida, já que todos os danos locais são modelados da mesma forma através de uma classe de objetos paramétrica, sem necessidades específicas de representação da geometria. Os elementos "patch" providenciam uma informação visual clara dos danos, mas simplificada, sendo o LoIN obtido essencialmente através de características não gráficas.

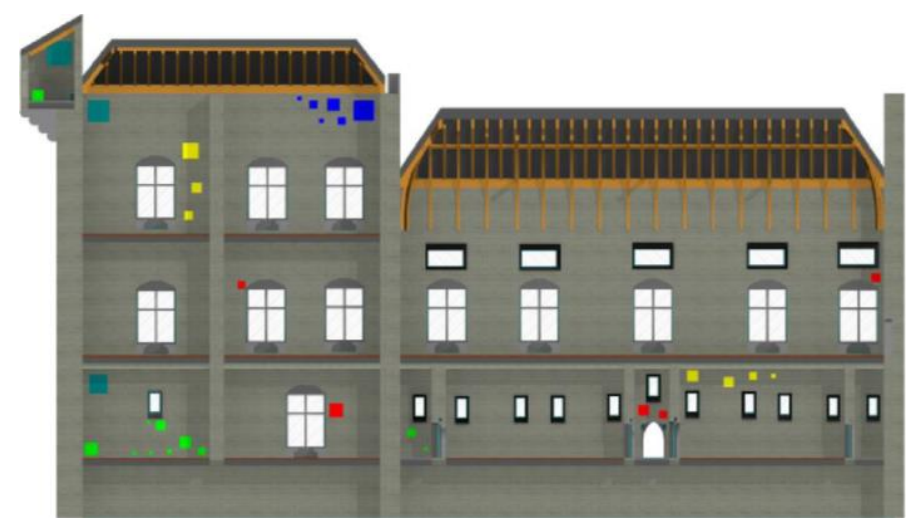

Figura 3: Levantamento da fachada Sul, com a dimensão dos elementos "patch" a representar a extensão dos danos. 
Os resultados do primeiro levantamento de danos foram atualizados no modelo usando um software proprietário, sendo posteriormente convertido para IFC. Nas inspeções seguintes, os profissionais envolvidos podem visualizar o IFC num software de acesso livre (Figura 3), que serve de índice para colocação de informação na folha de Excel desenvolvida para recolha. Cada elemento "patch" pode ser inquirido e detém uma identificação única através de um código que refere à sua folha de cálculo. Uma rápida alteração e atualização do modelo, baseada nas inspeções seguintes, é assim permitida assegurando a interoperabilidade entre software envolvidos. Em particular, cada pedaço de informação, recolhida no seu campo da folha de cálculo respetiva, tem de estar relacionada com um parâmetro tipo do software proprietário e daquele de livre acesso. Este processo é simplificado através da definição de tabelas de mapeamento de dados que ajudam a comparar em paralelo o tipo de parâmetro e o tipo de dados usados pelo software para descrever cada característica do PDT. A Tabela 3 apresenta um exemplo de mapeamento de dados para um dos elementos "patch", nomeadamente para fissuras estruturais profundas.

Tabela 3: Mapeamento de dados (Formato de dados / Revit / IFC), elemento dano, fissura estrutural profunda.

\begin{tabular}{|c|c|c|c|c|c|}
\hline $\begin{array}{l}\text { Parameter } \\
\text { Name }\end{array}$ & $\begin{array}{c}\text { Revit Group } \\
\text { Parameter / } \\
\text { Parameter } \\
\text { Name }\end{array}$ & $\begin{array}{c}\text { Revit } \\
\text { Type of } \\
\text { Parameter }\end{array}$ & $\begin{array}{l}\text { Revit } \\
\text { Data } \\
\text { Type }\end{array}$ & $\begin{array}{l}\text { IFC Property Set / } \\
\text { Parameter Name }\end{array}$ & $\begin{array}{c}\text { IFC } \\
\text { Data Type }\end{array}$ \\
\hline \multicolumn{6}{|c|}{ Classification Data } \\
\hline $\begin{array}{l}\text { Class of } \\
\text { Damage }\end{array}$ & Class of Damage & Type & Text & $\begin{array}{l}\text { HBIM_Classification } \\
\text { Data/Class of Damage }\end{array}$ & IFCTEXT \\
\hline $\begin{array}{l}\text { Sub-Sub-Class } \\
\text { of Damage }\end{array}$ & $\begin{array}{l}\text { Sub-Sub-Class of } \\
\text { Damage }\end{array}$ & Instance & Text & $\begin{array}{c}\text { HBIM_Classification } \\
\text { Data/Sub-Sub-Class of } \\
\text { Damage }\end{array}$ & IFCTEXT \\
\hline \multicolumn{6}{|c|}{ Inspection Data } \\
\hline $\begin{array}{l}\text { Inspection } \\
\text { Dates }\end{array}$ & $\begin{array}{l}\text { Construction / } \\
\text { Inspection } \\
\text { Dates }\end{array}$ & Instance & Text & $\begin{array}{c}\text { HBIM_Inspection } \\
\text { Data/ Inspection } \\
\text { Dates }\end{array}$ & IFCTEXT \\
\hline $\begin{array}{l}\text { Last Inspection } \\
\text { Inspector } \\
\text { reference }\end{array}$ & $\begin{array}{l}\text { Construction / } \\
\text { Last Inspection } \\
\text { Inspector } \\
\text { reference }\end{array}$ & Instance & Text & $\begin{array}{l}\text { HBIM_Inspection } \\
\text { Data/Last Inspection } \\
\text { Inspector reference }\end{array}$ & IFCTEXT \\
\hline $\begin{array}{c}\text { Brief } \\
\text { Description }\end{array}$ & $\begin{array}{l}\text { Identity Data / } \\
\text { Description }\end{array}$ & Type & Text & $\begin{array}{c}\text { HBIM_Inspection } \\
\text { Data/Brief Description }\end{array}$ & IFCTEXT \\
\hline $\begin{array}{l}\text { Survey Picture } \\
\text { URL }\end{array}$ & $\begin{array}{l}\text { Identity Data / } \\
\text { Image }\end{array}$ & Instance & File & $\begin{array}{c}\text { HBIM_Inspection } \\
\text { Data/Survey Picture } \\
\text { URL }\end{array}$ & IFCLABEL \\
\hline \multicolumn{6}{|c|}{ Geometric data } \\
\hline Area & Dimension/Area & Instance & Area & & \\
\hline Depth & Dimension/Depth & Instance & Length & $\begin{array}{c}\text { HBIM_Geometric Data/ } \\
\text { Depth }\end{array}$ & $\begin{array}{l}\text { IFC LENGTH } \\
\text { MEASURE }\end{array}$ \\
\hline Pattern & Dimension/Pattern & Instance & Text & $\begin{array}{c}\text { HBIM_Geometric Data/ } \\
\text { Pattern }\end{array}$ & IFCTEXT \\
\hline Form & Dimension/Form & Instance & Text & $\begin{array}{c}\text { HBIM_Geometric Data/ } \\
\text { Form }\end{array}$ & IFCTEXT \\
\hline
\end{tabular}




\begin{tabular}{|c|c|c|c|c|c|}
\hline \multicolumn{6}{|c|}{ Symptoms and Diagnosis } \\
\hline Possible Causes & Other/Possible Causes & Type & Text & $\begin{array}{c}\text { HBIM_Symptoms and } \\
\text { Diagnosis/Possible } \\
\text { Causes }\end{array}$ & IFCTEXT \\
\hline $\begin{array}{c}\text { Possible } \\
\text { Consequences }\end{array}$ & $\begin{array}{l}\text { Other/Possible } \\
\text { Consequences }\end{array}$ & Type & Text & $\begin{array}{c}\text { HBIM_Symptoms and } \\
\text { Diagnosis/Possible } \\
\text { Consequences }\end{array}$ & IFCTEXT \\
\hline Condition grade & Other/Condition grade & Type & Integer & $\begin{array}{c}\text { HBIM_Symptoms and } \\
\text { Diagnosis/Condition } \\
\text { grade }\end{array}$ & $\begin{array}{l}\text { IFC COUNT } \\
\text { MEASURE }\end{array}$ \\
\hline $\begin{array}{c}\text { Condition } \\
\text { Classification }\end{array}$ & $\begin{array}{l}\text { Other/Condition } \\
\text { Classification }\end{array}$ & Type & Text & $\begin{array}{l}\text { HBIM_Symptoms and } \\
\text { Diagnosis/Condition } \\
\text { Classification }\end{array}$ & IFCTEXT \\
\hline Symptoms & Other/Symptoms & Type & Text & $\begin{array}{c}\text { HBIM_Symptoms and } \\
\text { Diagnosis/Symptoms }\end{array}$ & IFCTEXT \\
\hline $\begin{array}{l}\text { Urgency Risk } \\
\text { Classification }\end{array}$ & $\begin{array}{c}\text { Other/Urgency Risk } \\
\text { Classification }\end{array}$ & Type & Text & $\begin{array}{c}\text { HBIM_Symptoms and } \\
\text { Diagnosis/Urgency Risk } \\
\text { Classification }\end{array}$ & IFCTEXT \\
\hline $\begin{array}{l}\text { Works Carried } \\
\text { Out }\end{array}$ & $\begin{array}{c}\text { Other/Works Carried } \\
\text { Out }\end{array}$ & Type & Text & $\begin{array}{c}\text { HBIM_Symptoms and } \\
\text { Diagnosis/Works Carried } \\
\text { Out }\end{array}$ & IFCTEXT \\
\hline \multicolumn{6}{|c|}{ Evolution Control Data } \\
\hline Type of Crack & Other/ Type of Crack & Instance & Text & $\begin{array}{c}\text { HBIM_Evolution } \\
\text { Control Data/ Type of } \\
\text { Crack }\end{array}$ & IFCTEXT \\
\hline Length & Other/ Length & Instance & Length & $\begin{array}{l}\text { HBIM_Evolution } \\
\text { Control Data/ Length }\end{array}$ & $\begin{array}{l}\text { IFC LENGTH } \\
\text { MEASURE }\end{array}$ \\
\hline Depth & Other/Depth & Instance & Length & $\begin{array}{l}\text { HBIM_Evolution } \\
\text { Control Data/ Depth }\end{array}$ & $\begin{array}{l}\text { IFC LENGTH } \\
\text { MEASURE }\end{array}$ \\
\hline Pattern & Other/Pattern & Instance & Text & $\begin{array}{c}\text { HBIM_Evolution } \\
\text { Control Data/ Pattern }\end{array}$ & IFCTEXT \\
\hline
\end{tabular}

\section{Conclusão}

Neste trabalho foi desenvolvida e testada uma metodologia baseada em BIM para inspeção e manutenção de construções históricas, contando com três objetivos principais: regulamentação de procedimentos, interoperabilidade dos componentes de software e simplificação de metodologias. Tendo em perspetiva estes objetivos gerais, o presente trabalho foca-se na descrição da abordagem desenvolvida, de fácil aplicabilidade, para o levantamento de danos e o acompanhamento da sua evolução ao longo do tempo. O nível de informação necessário para esta tarefa é atingido combinando informação gráfica e não gráfica, sem pretender uma representação extremamente precisa dos elementos estruturais reais e suas patologias. A geometria do edifício é representada com base em documentos existentes e inspeções tradicionais. O modelo é validado e potencialmente melhorado com a aquisição de uma nuvem de pontos obtida por varrimento laser. Cada elemento, por si próprio, age com um coletor de informação relevante e estabelece um sistema de referência, baseado na sua real geometria e posição no edifício. Nesse contexto, uma descrição mais exata da geometria dos elementos e 
dos danos foi considerada prejudicial para a praticabilidade do modelo e, portanto, não foi adotada. Todos os danos localizados são representados por elementos "patch" que são justapostos à superfície de cada elemento no local exato dos danos reais. A dimensão desses elementos muda conforme a extensão real do dano e a sua cor indica a classe, sendo esta baseada numa lista de danos pré-estabelecida na literatura como o caso do projeto HeritageCare. Todas as características relevantes do dano são acopladas a esse elemento "patch". Tal representação simbólica dos danos locais é inequívoca, consistente e, como tal, passível de ser adotada para regulamentação. Para além dessas características, é uma abordagem fácil de ser implementada e adaptada tendo em conta a natureza paramétrica dos elementos "patch". Finalmente, todos os procedimentos aqui descritos foram testados num caso de estudo, nomeadamente o Paço dos Duques de Bragança em Guimarães, um monumento relevante no contexto português, demonstrando-se a viabilidade e capacidade das metodologias e abordagens propostas.

\section{Agradecimentos}

Este trabalho foi parcialmente financiado pelo projeto HeritageCare (programa InterregSudoe/FEDER, SOE1/P5/P0258), por fundos do FEDER (programa COMPETE) e por fundos nacionais através da Fundação para a Ciência e Tecnologia FCT no âmbito do projeto POCI01-0145-FEDER-007633. Os autores agradecem o apoio da Dra. Isabel Fernandes, Diretora do Paço dos Duques de Bragança, pela permissão de acesso livre ao Paço. Agradece-se também a contribuição da TopArcos na realização do levantamento laser scanning em condições especiais para este trabalho de investigação da Universidade do Minho.

\section{Referências}

[1] Historic England, BIM for Heritage: Developing a Historic Building Information Model, International Society for Photogrammetry and Remote Sensing (ISPRS), 2017. https://content.historicengland.org.uk/images-books/publications/bim-for-heritage/heag154-bimfor-\%0Aheritage.pdf/.

[2] C. Brookes, The Application of Building Information Modelling (BIM) within a Heritage Science Context, 2017. http://research.historicengland.org.uk/redirect.aspx?id=6747\%7CThe Application of Building Information Modelling (BIM) within a Heritage Science Context.

[3] ISO 19650-1:2018 Organization and digitization of information about buildings and civil engineering works, including building information modelling (BIM) - Information management using building information modelling - Part 1: Concepts and principles, 2018.

[4] ISO 19650-2:2018 Organization and digitization of information about buildings and civil engineering works, including building information modelling (BIM) - Information management using building information modelling - Part 2: Delivery phase of the assets, 2018.

[5] G. Katie, Level of detail, information and accuracy in building information modelling of existing and heritage buildings, J. Cult. Herit. Manag. Sustain. Dev. 8 (2018) 495-507. 
[6] S. Garagnani, A.M. Manferdini, Parametric accuracy: building information modeling process applied to the cultural heritage preservation, Int. Arch. Photogramm. Remote Sens. Spat. Inf. Sci. 5 (2013).

[7] R. Brumana, S. Della Torre, M. Previtali, L. Barazzetti, L. Cantini, D. Oreni, F. Banfi, Generative HBIM modelling to embody complexity (LOD, LOG, LOA, LOI): surveying, preservation, site intervention - the Basilica di Collemaggio (L'Aquila), Appl. Geomatics. 10 (2018) 545-567.

[8] E. Fateeva, V. Badenko, A. Fedotov, I. Kochetkov, System analysis of the quality of meshes in HBIM, MATEC Web Conf. 170 (2018).

[9] F. Chiabrando, M. Lo Turco, F. Rinaudo, MODELING THE DECAY IN AN HBIM STARTING FROM 3D POINT CLOUDS. A FOLLOWED APPROACH FOR CULTURAL HERITAGE KNOWLEDGE., Int. Arch. Photogramm. Remote Sens. Spat. Inf. Sci. 42 (2017).

[10]M. Lo Turco, M. Mattonea, F. Rinaudoa, Metric survey and BIM technologies to record decay conditions, Int. Arch. Photogramm. Remote Sens. Spat. Inf. Sci. 42 (2017).

[11] R. Zeibak-Shini, R. Sacks, L. Ma, S. Filin, Towards generation of as-damaged BIM models using laser-scanning and as-built BIM: First estimate of as-damaged locations of reinforced concrete frame members in masonry infill structures, Adv. Eng. Informatics. 30 (2016) $312-326$.

[12]M.G. Masciotta, M.J. Morais, L.F. Ramos, D. V Oliveira, L.J. Sánchez-Aparicio, D. González-Aguilera, A Digital-based Integrated Methodology for the Preventive Conservation of Cultural Heritage: The Experience of HeritageCare Project, Int. J. Archit. Herit. (2019) 1-20.

[13]CIBSE, BIM - Building Information Modelling, (2017). https://www.cibse.org/knowledge/bim\%0A-\%0Abuilding\%0A-\%0Ainformation\%0A$\% 0$ Amo\%0Adelling/product\%0A-\%0Adata\%0A-\%0Atemplates (accessed June 11, 2018).

[14]C.A.B. Alarcón, Building information modelling in the context of structural preservation of historical monuments: methodological aspects and application to "Paço dos Duques"., University of Minho,Advanced Masters in Structural Analysis of Monuments and Historical Constructions, 2018.

[15] Direcção-Geral dos Edifícios e Monumentos Nacionais, Paço dos Duques de Bragança: Guimarães, Boletim da, Lisbon, 1960. 